\title{
GSTM1 polymorphism modifies the effect of smoking on calcaneal quantitative ultrasound in young men
}

\author{
TAO LIU ${ }^{1,2}$, XUECHUAN SUN ${ }^{1,2}$, XIAO ZHUANG ${ }^{3}$ and JINGQUN LIANG ${ }^{2}$ \\ ${ }^{1}$ West China School of Preclinical and Forensic Medicine, Sichuan University, Chengdu, Sichuan; \\ ${ }^{2}$ Scientific Research Department, PLA Institute of Physical Education, Guangzhou, Guangdong; \\ ${ }^{3}$ Physics Department, Medical College of Shantou University, Shantou, Guangdong, P.R. China
}

Received July 25, 2012; Accepted November 13, 2012

DOI: $10.3892 / \mathrm{mmr} .2012 .1192$

\begin{abstract}
Evidence suggests that smoking adversely affects bones. Glutathione S-transferases (GSTs) are important in the detoxification of tobacco smoke compounds. This study investigated the influence of GSTMI and GSTT1 genetic polymorphisms on the effects on bone induced by tobacco smoking in young men. In total, 231 Chinese men aged 20-39 years were assessed by a cross-sectional study. Calcaneal quantitative ultrasound (QUS) was measured. Smoking information was obtained using a questionnaire. Genotypes were determined for null alleles of GSTM1 and GSTT1. Smoking was inversely associated with speed of sound (SOS) and bone quality index (BQI) among subjects with GSTM1 null genotype $(\beta=-0.28,95 \% \mathrm{CI},-34.01$ to -8.65 and $\beta=-0.30,95 \%$ CI, -16.41 to -4.49 , respectively), but not among those with the allele present $(\beta=-0.02,95 \% \mathrm{CI},-15.01$ to 12.59 and $\beta=$ $-0.09,95 \% \mathrm{CI},-8.75$ to 3.02 , respectively). $\mathrm{P}$ for interaction $=0.04$ for SOS and 0.08 for BQI. No interaction was found between the GSTT1 polymorphism and smoking. The results showed that GSTM1 deficiency may increase the adverse effect of smoking on calcaneal QUS in young men, suggesting an interaction between metabolic genes and tobacco smoke in bone detoxification.
\end{abstract}

\section{Introduction}

There is now ample evidence to suggest that smoking adversely affects bones. The bone mass achieved in young

Correspondence to: Professor Xuechuan Sun, West China School of Preclinical and Forensic Medicine, Sichuan University, Chengdu, Sichuan 610041, P.R. China

E-mail:wininky@yeah.net

Abbreviations: BMD, bone mineral density; GST, glutathione S-transferase; QUS, quantitative ultrasound; BMI, body mass index; BUA, broadband ultrasound attenuation; SOS, speed of sound; BQI, bone quality index

Key words: bone, smoking, glutathione S-transferase, polymorphism, interaction, young men adults is of importance for bone density in later life (1). Thus, although the majority of osteoporotic fractures occur in elderly people, the risk may be profoundly affected by events in early life. In young men, the adverse effects of smoking have been demonstrated in both bone mass and bone morphology. Ortego-Centeno et al (2) reported that smoking by healthy young males aged 20-45 years is associated with decreased bone mineral density (BMD). A prospective cohort study showed that not smoking is important in achieving maximum peak bone mass in adolescents and young adults (20-29 years) (3). Lorentzon et al (4) observed a $4-5 \%$ decrease in femoral neck and lumbar spine areal BMD and in distal tibia volumetric BMD and cortical tibial area in healthy young male smokers compared with nonsmokers, confirming that smoking affects bone at the tissue level. Similar results are obtained for the radius of men aged $\sim 40$ years (5). The adverse effects of smoking on bone mass and bone morphology appear to be more pronounced in men than in women $(3,6)$.

Although smoking is considered as a threat to bone health, the meaning of the association and the mechanism remain poorly understood. Tobacco smoke contains $>4000$ compounds. The metabolism and detoxification of tobacco smoke toxins are essential mechanisms to decrease the adverse effects of smoking. Glutathione S-transferases (GSTs) are an important superfamily of phase II xenobiotic metabolizing enzymes involved in a wide range of these detoxification processes (7). Certain genetic variants of GSTs have been identified. The most commonly investigated variants include the complete deletions (null polymorphisms) of the GSTM1 and GSTT1 genes. GSTM1 and GSTT1 homozygous deletions (null genotypes) result in the absence of the respective functional enzyme activity $(8,9,10)$. Moreover, GSTM1 is absent in $35-60 \%$ of the human population, and GSTT1 is absent in $10-65 \%$ of individuals $(11,12)$. Thus, GSTM1 or GSTT1 null polymorphism may have a greater effect on health.

The aim of this study was to examine whether the association between tobacco smoking and bone status, as assessed by calcaneus quantitative ultrasound (QUS), differs according to GSTM1 or GSTT1 null polymorphisms in young men. An interaction with genes involved in tobacco smoke detoxification would support the toxicity effect of smoking on bone and help us to understand the related mechanisms. 


\section{Subjects and methods}

Subjects. In total, 250 men were randomly selected from the male residents aged 20-39 years of the Chancheng District, Foshan City, Guangdong Province, China, who participated in the Foshan Public Physique Monitoring Program in 2005. The study was initiated with the aim of determining genetic factors involved in bone health. Among these men, 9 were unwilling to provide blood samples, and therefore were not included in this study. Men who had diseases (2 reporting kidney stone and 1 reporting forearm fracture surgery) or drug treatments (4 reporting a long-term calcium supplementation) known to affect bone were excluded. Of the eligible men, 3 reporting never smoking but having long-term passive smoking were also excluded. As a result, 231 participants were included in the present analyses. All subjects were unrelated Han Chinese. Written informed consent was obtained. The study was carried out according to the principles of the Declaration of Helsinki.

Study variables. Information on smoking status was obtained by interviewer-administered questionnaires. The subjects were classified into three groups: i) subjects reporting no smoking in their lifetime; ii) subjects reporting occasional smoking ( $\leq 20$ cigarettes per year); and iii) subjects reporting current smoking. The current smokers smoked at least 5 cigarettes per day for $\geq 2$ years, with no periods of smoking cessation for $>1$ week in the 2 years prior to this investigation. No ex-smokers were found in the present study. As the number of occasional smokers was small and they consumed few cigarettes, groups i) and ii) were combined into non-smokers.

Physical activity was assessed using the Baecke questionnaire (13), and a value of $<7$ represented a low physical activity level. Body weight and height were measured with the subjects dressed in lightweight clothing without shoes. Body mass index (BMI) was calculated as body weight in kilograms divided by the square of height in meters.

QUS measurements at the left calcaneus were taken using a SONOST-2000 Bone Densitometer (Osteosys, Seoul, South Korea) by a trained technician. Outputs included broadband ultrasound attenuation (BUA), speed of sound (SOS), and bone quality index (BQI) derived from linear combinations of SOS and BUA. Short-term in vivo precisions were established on the basis of 150 measurements in 15 subjects (10 measurements each). The precision errors were $1.5 \%$ for BUA, $0.2 \%$ for SOS and $1.5 \%$ for BQI. Daily quality assurance tests of the QUS device, as recommended by the manufacturers, were taken to monitor system stability. To reduce the effect of temperature, all measurements were conducted in a controlled environment at $25^{\circ} \mathrm{C}$. The QUS measurement of each subject was taken twice, and the average was used in analyses. All the measurements were taken by the same QUS device and technician. The technician performing the QUS testing did not know the smoking status of the subjects.

Genotyping. Genomic DNA was extracted from the subjects' peripheral blood leukocytes using a standard phenol-chloroform extraction procedure, and samples were stored at $-20^{\circ} \mathrm{C}$.

GSTM1 and GSTT1 genes were detected simultaneously in a single assay by the multiplex PCR approach, as described previously (14). PCR products were then separated by electrophoresis on a $1.5 \%$ agarose gel. GSTM1 and GSTT1 genotypes were determined by the presence or absence of bands at 480 and 215 bp, respectively. The absence of a GSTM1 or GSTT1 band indicated the corresponding null genotype (homozygous deletion). A band at $312 \mathrm{bp}$ (corresponding to exon 7 of CYP1A1 gene) was always present and was used as an internal control to demonstrate successful PCR amplification. All assays were conducted without being aware of the QUS outcomes and smoking status.

Statistical analysis. Continuous variables were expressed as the means $\pm \mathrm{SD}$, and categorical variables as numbers and percentages. Age, BMI and BUA were logarithmically transformed to reduce skewness. Comparisons of variables between groups were performed with the Student's t-test and Chi-square test, and the relationship between continuous variables was assessed by correlation analyses. Linear regression models were performed to examine the association between smoking status and QUS outcomes according to GSTM1 or GSTT1 genotype. Age and physical activity were selected as confounding variables, and retained in the final model if they modified coefficients of smoking by $\geq 10 \%$. The interactions between smoking and GSTM1 and GSTT1 genes were assessed by including interaction terms. All analyses were performed using SPSS version 13.0 (SPSS, Chicago, IL, USA).

\section{Results}

In our study population, the frequencies of GSTM1 and GSTT1 null genotypes were 54.1 and $48.1 \%$, respectively. The prevalence of smoking was $36.4 \%$. Age and physical activity were significantly associated with QUS parameters $(\mathrm{P}<0.03)$ (data not shown). However, we did not detect an association of BMI with QUS outcomes ( $\mathrm{P}>0.2)$ (data not shown). Smokers had lower BUA $(\mathrm{P}=0.001)$, SOS $(\mathrm{P}=0.01)$ and BQI $(\mathrm{P}=0.001)$ compared with non-smokers (data not shown).

In the population, the genotypic distribution of GSTM1 or GSTT1 did not differ according to smoking status, and GSTM1 or GSTT1 polymorphisms were not associated with QUS outcomes (Table I).

After adjusting for confounding variables, smoking was inversely associated with SOS and BQI $(\beta=-0.28,95 \% \mathrm{CI}$ -34.01 to -8.65 and $\beta=-0.30,95 \% \mathrm{CI},-16.41$ to -4.49 , respectively) among subjects with a null genotype for GSTM1, but not among subjects with the allele present $(\beta=-0.02,95 \% \mathrm{CI}$ -15.01 to 12.59 and $\beta=-0.09,95 \% \mathrm{CI},-8.75$ to 3.02 , respectively) ( $\mathrm{P}$ for interaction $=0.04$ for $\mathrm{SOS}$ and 0.08 for $\mathrm{BQI}$ ) (Table II). No interaction between smoking and GSTT1 polymorphism was observed (Table II).

\section{Discussion}

In the present study, GSTM1 null polymorphism modified the association between smoking and calcaneus QUS in young men. Individuals with the GSTM1 null genotype who had a smoking habit were more at risk of adverse QUS. These results support the toxic effect of smoking on bone in young men. Although our study population was small, the interaction between smoking and GSTM1 polymorphism suggested in the study adds to the plausibility of a biological 
Table I. Smoking status and QUS outcomes according to GSTM1 and GSTT1 genotypes in the study population (n=231).

\begin{tabular}{|c|c|c|c|c|c|c|}
\hline \multirow[b]{2}{*}{ Variables } & \multicolumn{3}{|c|}{ GSTM1 genotypes } & \multicolumn{3}{|c|}{ GSTT1 genotypes } \\
\hline & Present $(n=106)$ & Null $(n=125)$ & P-value ${ }^{a}$ & Present $(n=120)$ & Null $(n=111)$ & P-value \\
\hline Smoking, n (\%) & & & $0.49^{\mathrm{b}}$ & & & $0.08^{\mathrm{b}}$ \\
\hline No & $70(66.1)$ & 77 (61.6) & & $70(58.3)$ & 77 (69.4) & \\
\hline Yes & $36(33.9)$ & $48(38.4)$ & & $50(41.7)$ & $34(30.6)$ & \\
\hline \multicolumn{7}{|c|}{ QUS outcomes, mean (SD) } \\
\hline $\mathrm{BUA}(\mathrm{dB} / \mathrm{MHz})$ & $52.8(12.1)$ & $53.3(12.6)$ & 0.77 & $53.3(12.7)$ & $52.7(12.1)$ & 0.73 \\
\hline $\operatorname{SOS}(\mathrm{m} / \mathrm{s})$ & $1561.7(36.6)$ & $1558.1(36.7)$ & 0.45 & $1559.1(35.1)$ & $1560.5(38.3)$ & 0.77 \\
\hline BQI & $95.6(15.8)$ & $95.1(17.2)$ & 0.79 & $95.2(16.5)$ & $95.4(16.7)$ & 0.94 \\
\hline
\end{tabular}

QUS, quantitative ultrasound; BUA, broadband ultrasound attenuation; SOS, speed of sound; BQI, bone quality index. ${ }^{a}$ Indicate P-value derived from the Student's t-test unless otherwise stated. ${ }^{\mathrm{b}} \mathrm{P}$-value derived from the Chi-square test.

Table II. Associations (coefficient, $\beta$ and 95\% CI) between QUS outcomes and smoking by GSTM1 and GSTT1 genotypes.

\begin{tabular}{|c|c|c|c|c|c|c|c|c|c|}
\hline \multirow[b]{2}{*}{ Genotypes } & \multicolumn{3}{|c|}{ BUA } & \multicolumn{3}{|c|}{ SOS } & \multicolumn{3}{|c|}{ BQI } \\
\hline & $\beta$ & $95 \% \mathrm{CI}$ & P-value ${ }^{a}$ & $\beta$ & $95 \% \mathrm{CI}$ & P-value ${ }^{a}$ & $\beta$ & $95 \%$ CI & P-value \\
\hline GSTM1 & & & 0.40 & & & 0.04 & & & 0.08 \\
\hline Present & -0.15 & -8.57 to 0.82 & & -0.02 & -15.01 to 12.59 & & -0.09 & -8.75 to 3.02 & \\
\hline Null & -0.26 & -11.11 to -2.17 & & -0.28 & -34.01 to -8.65 & & -0.30 & -16.41 to -4.49 & \\
\hline GSTT1 & & & 0.98 & & & 0.91 & & & 0.97 \\
\hline Present & -0.22 & -10.23 to -1.01 & & -0.17 & -25.43 to -0.09 & & -0.22 & -13.14 to -1.32 & \\
\hline Null & -0.21 & -10.43 to -0.71 & & -0.14 & -26.08 to 3.56 & & -0.19 & -13.43 to -0.45 & \\
\hline
\end{tabular}

QUS, quantitative ultrasound; BUA, broadband ultrasound attenuation; SOS, speed of sound; BQI, bone quality index. Coefficients were calculated by linear regression models, adjusted for age and physical activity. For all comparisons, the reference category is no smoking.

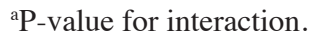

interaction between GSTs and tobacco smoke components in bone detoxification.

In this study, GSTM1 and GSTT1 genotype distributions are comparable with those previously reported in other normal Chinese populations (15). GSTM1 and GSTT1 null variants are prevalent in the human population $(11,12)$. Common genetic variants of relevant detoxification enzymes may determine the individual susceptibility to environmental toxicants. In particular, the GSTMI null genotype has been identified as a susceptibility factor of common smokingrelated cancers, respiratory and cardiovascular diseases. To the best of our knowledge, this is the first study to assess the influence of common genetic polymorphisms on the effects on bone induced by smoking. Tracking down the genetic polymorphisms that regulate metabolic pathways of relevance to environmental exposures and diseases may provide better understanding of causation.

We did not find an interaction between smoking and GSTT1 genotype in this study. The reason for this is not clear. Although GSTT1 is predominantly involved in the detoxification process of xenobiotics, certain toxic compounds may be activated through the GSTT1 pathway (16). Similar to our results, previous studies on diseases did not observe an interaction between GSTT1 null polymorphism and smoking, but reported the modification effect of GSTM1 $(17,18)$.

GSTs are important in the metabolism processes of many toxic compounds contained in cigarette smoke, and therefore may have a function in the protection of bone. However, which components of cigarette smoke adversely affect bone remains unclear. Nicotine may be involved in the toxic process. In vitro studies have shown decreased proliferation and impaired collagen synthesis in osteoblast-like cells exposed to high nicotine concentration $(19,20)$. The adverse effect of nicotine is further supported by a study on adult rats demonstrating that nicotine may adversely affect bone formation and reduce body storage of vitamin D (21). Another explanation for the mechanism by which GSTs protect bone against smoking may be related to their important roles in defending against oxidative stress (22). It is well-known that smoking increases the level of oxidative stress. Increased oxidative stress is associated with reduced bone density (23). Increased reactive oxygen species stimulate osteoclast formation and activity, and therefore enhance bone resorption (24). Moreover, elevated reactive oxygen species suppress osteoblast differentiation and 
promote osteoblast apoptosis (25). Oxidative stress may be a pivotal pathogenetic mechanism of bone loss and strength attenuation (26). Individuals with GSTM1 null genotype, due to the absence of corresponding functional enzyme activity, may therefore be more susceptible to the toxic effect of tobacco smoke exposure.

Certain potential limitations in the present study should be considered and our results should be interpreted with caution. First, this was a cross-sectional study and our study population was small in size. Furthermore, our analyses have a potential problem of multiple comparisons. Therefore, subsequent large and prospective studies are required to confirm our results. The gene-gene interaction was not analysed in view of the small sample sizes in the present study. Second, we did not have data from all selected men, which made selection bias possible. Third, we cannot exclude the possibility that our results may be confounded by other genetic variants that are in linkage disequilibrium with the present variant. Certain other confounding factors, such as calcium intake, were not included in this study. In addition, we did not use dual-energy X-ray absorptiometry or peripheral quantitative computed tomography, which may be better methods in certain respects, to assess bone.

In conclusion, GSTM1 deficiency may increase the adverse effect of smoking on bone, as shown by calcaneus quantitative ultrasound in young men, suggesting an interaction between metabolic genes and tobacco smoke in bone detoxification.

\section{Acknowledgements}

This study was supported by the Foshan Foundation for Development of Science and Technology (200508089). We thank the volunteers who gave their time and blood samples for this investigation. We would like to thank Weihong Bo (Foshan National Physique Monitoring Center) and Bin $\mathrm{Hu}$ (Da An Gene Diagnosis Center of Sun Yat-sen University) for their technological support.

\section{References}

1. Valimaki MJ, Karkkainen M, Lamberg-Allardt C, et al: Exercise, smoking, and calcium intake during adolescence and early adulthood as determinants of peak bone mass. Br Med J 309: 230-235, 1994.

2. Ortego-Centeno N,Munoz-Torres M,JodarE,Hernandez-Quero J, Jurado-Duce A and Torres-Puchol JH: Effect of tobacco consumption on bone mineral density in healthy young males. Calcif Tissue Int 60: 496-500, 1997.

3. Kelly PJ, Morrison NA, Sambrook PN, Nguyen TV and Eisman JA: Genetic influences on bone turnover, bone density and fracture. Eur J Endocrinol 133: 265-271, 1995.

4. Lorentzon M, Mellström D, Haug E and Ohlsson C: Smoking is associated with lower bone mineral density and reduced cortical thickness in young men. J Clin Endocrinol Metab 92: 497-503, 2007.

5. Medraœ M, Jankowska EA and Rogucka E: The effect of smoking tobacco and drinking of alcohol and coffee on bone mineral density of healthy men 40 years of age. Pol Arch Med Wewn 103: 187-193, 2000.

6. Wüst RCI, Winwood K, Wilks DC, Morse CI, Degens H and Rittweger J: Effects of smoking on tibial and radial bone mass and strength may diminish with age. J Clin Endocrinol Metab 95: 2763-2771, 2010.
7. Sheehan D, Meade G, Foley VM and Dowd CA: Structure, function and evolution of glutathione transferases: implications for classification of non-mammalian members of an ancient enzyme superfamily. Biochem J 360: 1-16, 2001.

8. Pemble S, Schroeder KR, Spencer SR, et al: Human glutathione S-transferase theta (GSTT1): cDNA cloning and the characterization of a genetic polymorphism. Biochem J 300: 271-276, 1994.

9. Seidegard J, Vorachek WR, Pero RW and Pearson WR: Hereditary differences in the expression of the human glutathione transferase active on trans-stilbene oxide are due to a gene deletion. Proc Natl Acad Sci USA 85: 7293-7297, 1988.

10. Dialyna IA, Miyakis S, Georgatou N and Spandidos DA: Genetic polymorphisms of CYP1A1, GSTM1 and GSTT1 genes and lung cancer risk. Oncol Rep 10: 1829-1835, 2003.

11. Setiawan VW, Zhang ZF, Yu GP, et al: GSTT1 and GSTM1 null genotypes and the risk of gastric cancer: a case-control study in a Chinese population. Cancer Epidemiol Biomarkers Prev 9: 73-80, 2000 .

12. Nelson HH, Wiencke JK, Christiani DC, et al: Ethnic differences in the prevalence of the homozygous deleted genotype of glutathione S-transferase $\theta$. Carcinogenesis (Lond) 16: 1243-1245, 1995.

13. Baecke JA, Burema J and Frijters JE: A short questionnaire for the measurement of habitual physical activity in epidemiological studies. Am J Clin Nutr 36: 936-942, 1982.

14. Abdel-Rahman SZ, el-Zein RA, Anwar WA and Au WW: A multiplex PCR procedure for polymorphic analysis of GSTM1 and GSTT1 genes in population studies. Cancer Lett 107: 229-233, 1996.

15. Shen J, Lin G, Yuan W, Tan J, Bolt HM and Thier R: Glutathione transferase T1 and M1 genotype polymorphism in the normal population of Shanghai. Arch Toxicol 72: 456-458, 1998.

16. Landi S: Mammalian class theta GST and differential susceptibility to carcinogens: a review. Mutat Res 463: 247-283, 2000.

17. Ruano-Ravina A, Figueiras A, Loidi L and Barros-Dios JM: GSTM1 and GSTT1 polymorphisms, tobacco and risk of lung cancer: a case-control study from Galicia, Spain. Anticancer Res 23: 4333-4337, 2003.

18. Lee KM, Kang D, Lee SJ, et al: Interactive effect of genetic polymorphism of glutathione S-transferase M1 and smoking on squamous cell lung cancer risk in Korea. Oncol Rep 16: 1035-1039, 2006

19. Fang MA, Frost PJ, Iida-Klein A and Hahn TJ: Effects of nicotine on cellular function in UMR 106-01 osteoblast-like cells. Bone 12: 283-286, 1991.

20. Ramp WK, Lenz LG and Galvin RJS: Nicotine inhibits collagen synthesis and alkaline phosphatase activity, but stimulates DNA synthesis in osteoblast-like cells. Proc Soc Exp Biol Med 197: 36-43, 1991.

21. Fung YK, Iwaniec U, Cullen DM, Akhter MP, Haven MC and Timmins P: Long-term effects of nicotine on bone and calciotropic hormones in adult female rats. Pharmacol Toxicol 85: 181-187, 1999.

22. Hayes JD and McLellan LI: Glutathione and glutathionedependent enzymes represent a co-ordinately regulated defence against oxidative stress. Free Radic Res 31: 273-300, 1999.

23. Basu S, Michaelsson K, Olofsson H, Johansson S and Melhus H: Association between oxidative stress and bone mineral density. Biochem Biophys Res Commun 288: 275-279, 2001.

24. Koh JM, Lee YS, Kim YS, et al: Homocysteine enhances bone resorption by stimulation of osteoclast formation and activity through increased intracellular ROS generation. J Bone Miner Res 21: 1003-1011, 2006.

25. Almeida M, Han L, Martin-Millan M, O'Brien CA and Manolagas SC: Oxidative stress antagonizes wnt signaling in osteoblast precursors by diverting $\beta$-catenin from $\mathrm{T}$ cell factorto forkhead box O-mediated transcription. J Biol Chem 282: 27298-27305, 2007.

26. Almeida M, Han L, Martin-Millan M, et al: Skeletal involution by age-associated oxidative stress and its acceleration by lossof sex steroids. J Biol Chem 282: 27285-27297, 2007. 